\title{
Carmustine as a Supplementary Therapeutic Option for Glioblastoma: A Systematic Review and Meta-Analysis
}

\author{
Zhi-Ze Xiao ${ }^{1 \dagger}$, Ze-Fen Wang ${ }^{2 \dagger}$, Tian Lan ${ }^{1}$, Wen-Hong Huang ${ }^{1}$, Yu-Hang Zhao ${ }^{1}$, Chao Ma ${ }^{1}$ \\ and Zhi-Qiang $\mathrm{Li}^{1,3 *}$
}

${ }^{1}$ Department of Neurosurgery, Zhongnan Hospital of Wuhan University, Wuhan, China, ${ }^{2}$ Department of Physiology, School of Basic Medical Sciences, Wuhan University, Wuhan, China, ${ }^{3}$ Laboratory of Neuro-Oncology, Zhongnan Hospital of Wuhan University, Wuhan, China

\section{OPEN ACCESS}

Edited by:

Adam M. Sonabend, Northwestern University, United States

Reviewed by:

Joerg Robert Leheste, Anoka-Ramsey Community College, United States

Dusten Unruh,

Northwestern Medicine, United States

*Correspondence: Zhi-Qiang Li

lizhiqiang@whu.edu.cn

tThese authors have contributed equally to this work

Specialty section:

This article was submitted to Neuro-Oncology and Neurosurgical Oncology,

a section of the journa Frontiers in Neurology

Received: 16 April 2020 Accepted: 10 August 2020 Published: 17 September 2020

Citation:

Xiao Z-Z, Wang Z-F, Lan T, Huang $W-H$, Zhao $Y-H, M a C$ and

Li Z-Q (2020) Carmustine as a Supplementary Therapeutic Option for Glioblastoma: A Systematic Review and Meta-Analysis.

Front. Neurol. 11:1036. doi: 10.3389/fneur.2020.01036
Background: Glioblastoma (GBM) is the most aggressive type of primary malignant brain tumor. Carmustine is used by intravenous injection or local implantation in the resection cavity for gliomas, including GBMs. However, the therapeutic potential of carmustine is not well-recognized. This analysis aimed to evaluate the survival benefits of carmustine in glioma patients, especially those with GBM.

Methods: Randomized controlled trials (RCTs) and cohort studies regarding carmustine for glioma treatment were searched in PubMed, the Cochrane Library, and Embase from January 1979 to March 2020. Quality assessment was conducted with Jadad and Newcastle-Ottawa scales (NOS). Statistical analysis was conducted by the Revman 5.3 software.

Results: Twenty-two eligible RCTs and cohort studies involving 5,821 glioma patients were included. Overall, glioma patients receiving carmustine as an adjuvant therapy had better progression-free survival $[\mathrm{PFS}$; hazard ratio $(\mathrm{HR})=0.85,95 \% \mathrm{Cl}=0.77-0.94$, $P=0.002$ ] and overall survival (OS; HR $=0.85,95 \% \mathrm{Cl}=0.79-0.92, P<$ 0.0001 ) than those without carmustine treatment. Subgroup analysis showed that the OS benefit was observed in GBM $(\mathrm{HR}=0.84,95 \% \mathrm{Cl}=0.78-0.91, P<$ $0.00001)$ but not in anaplastic glioma patients $(H R=1.20,95 \% \mathrm{Cl}=0.70-2.07$, $P=0.50$ ). Additionally, both newly diagnosed and recurrent GBM patients who received carmustine treatment showed better OS $(\mathrm{HR}=0.86,95 \% \mathrm{Cl}=0.79-0.95$, $P=0.002 ; \mathrm{HR}=0.77,95 \% \mathrm{Cl}=0.67-0.89, P=0.0002$, respectively). Both carmustine implantation in resection cavity and intravenous administration significantly prolonged OS $(\mathrm{HR}=0.84,95 \% \mathrm{Cl}=0.78-0.92, P<0.0001 ; \mathrm{HR}=0.86,95 \% \mathrm{Cl}=0.75-0.99$, $P=0.04$, respectively). Moreover, GBM patients receiving a combined carmustine and temozolomide (TMZ) therapy had longer OS than those receiving $T M Z$ alone $(H R=0.78$, $95 \% \mathrm{Cl}=0.63-0.97, P=0.03)$.

Conclusion: Carmustine implantation in resection cavity provides survival benefit for GBM patients, and it may be a promising supplement to standard therapeutic protocol by offering a bridge between surgical resection and onset of TMZ therapy.

Keywords: glioblastoma, carmustine (BCNU), overall survival (OS), meta-analysis, chemotherapy 


\section{INTRODUCTION}

Glioma is the most common primary malignant central nervous system tumor. Glioblastoma (GBM), World Health Organization (WHO) grade 4 glioma subtype, is one of the most deadly malignant tumors with an estimated incidence of 5.26 per 100,000 population or 17,000 new diagnosed patients per year (1). GBMs are present at the median age of 64 years, but can occur at any age, including in children (2). The standard care for newly diagnosed GBM is maximal safe surgical resection, followed by radiochemotherapy with the alkylating agent, temozolomide (TMZ). However, the tumor inevitably recurs, and standardized strategies for the treatment of recurrent glioma are lacking. The evidences of a favorable outcome regarding re-resection and re-irradiation are still poor (3). Therefore, systemic chemotherapy has been explored as a prospective option for glioma.

The nitrosourea derivative, carmustine $\{\mathrm{BCNU},[1,3$-bis (2chloroethyl)-1-nitrosourea]\}, another alkylating agent, has also been used both at the initial diagnosis of glioma and at tumor recurrence, either by intravenous administration or by wafer implantation. Biodegradable wafers impregnated with carmustine (Gliadel ${ }^{\circledR}$ wafer) and implanted in the surgical bed on the walls of the resection cavity (4) were developed initially by Brem et al. to avoid the toxicity associated with systemic administration of carmustine (5). Carmustine wafer implantation in GBM patients undergoing surgical resection is also thought to provide a therapeutic bridge during the period between surgical resection and onset of radiotherapy (6). Previous research assessing the effectiveness of carmustine wafer has found a significant increase in overall survival (OS) by 2-4 months in newly diagnosed GBM patients (3). Although the efficacy of carmustine administration is established in seminal trials, its safety remains controversial. The common side effects of carmustine-based chemotherapy include nausea/vomiting and hematotoxicity with a delayed nadir after 4-6 weeks, and the most dreaded side effect, pulmonary fibrosis. For carmustine wafer implantation, its impact on post-operative infections, quality of life, and feasibility of adjuvant oncological treatments has also been debatable (6). The experimental data of carmustine administration are rather controversial, and there is no general agreement about adverse events. A systematic understanding on whether carmustine wafer contributes to survival in glioma patients is still lacking.

This study aimed to assess whether carmustine treatment is beneficial for GBM and other gliomas, and whether the addition of carmustine therapy as a supplement to STUPP protocol could provide more survival benefits for GBM patients.

Abbreviations: GBM, glioblastoma; Gliadel ${ }^{\circledR}$ wafer, carmustine; BCUN, N,N' bis(2-chloroethyl)-N-nitrosourea; TMZ, temozolomide; RCTs, randomized controlled trials; NOS, Newcastle-Ottawa scales; PFS, progression-free survival; OS, overall survival; KPS, Karnofsky performance score.

\section{METHODS}

\section{Search Strategy}

A literature search was performed by two independent reviewers across three databases including PubMed, Embase, and the Cochrane Library. Randomized controlled trials (RCTs) and cohorts involving carmustine for glioma treatment were retrieved by searching databases from January 1979 to March 2020. For the search, the following string of terms was used: (astrocytoma OR oligodendroglioma OR oligodendroglial OR glioma OR glioblastoma) AND [carmustine OR BCNU OR N, $N^{\prime}$ bis (2-chloroethyl)-N-nitrosourea OR 1,3-Bis (2-chloroethyl)1-nitrosourea OR FIVB (fluorouracil, imidazole carboxamide dimethyl triazeno, vincristine, and bis-chloroethyl nitrosourea) OR BCNU OR Nitrumon OR carmustine wafers OR Gliade]. Reviews and references of included studies were also checked to avoid omission of relevant publications. The studies were restricted to human beings.

\section{Selection Criteria}

RCTs and cohort researches meeting the following criteria were considered eligible: (1) RCTs regarding carmustine treatment in glioma patients irrespective of blinding methods and publishing language, and cohort studies regarding carmustine treatment in glioma patients; (2) studies with comparison of therapeutic regimen with or without carmustine; and (3) studies containing the hazard ratios (HRs) for OS, progression-free survival (PFS) or survival curves, or details from computed data presented.

RCTs and cohort studies meeting the following criteria were excluded in this meta-analysis: (1) duplicate reports or studies lacking adequate original data and (2) studies in which patients suffered from other primary tumors, trauma, or severe infections.

\section{Data Extraction and Quality Assessment}

Two researchers read the titles and abstracts of the identified literature, excluding the irrelevant ones, reviews, and pharmacological experiments. Full texts of the possibly pertinent RCTs and cohort studies were checked further to determine whether they fulfilled the inclusion criteria. The reference sections of the retrieved articles were also screened. Case reports, animal experiments, editorials, and letters were excluded. If the eligible studies provided both univariate and multivariate analyses, hazard ratios (HRs) calculated by multivariate analysis were preferred as these values had higher precision for interpreting confounding factors in the Cox regression model. The two researchers conducted their quality evaluations independently. If there were disagreements, decisions were made following discussions or further inquiry by a third researcher.

RCTs using the Jadad scale (ranging from 0 to 5) were considered to be of high quality (7). The Newcastle-Ottawa scale (NOS) was used to assess the quality of the included cohort studies (8). There were three main aspects including study selection ( $0-4$ points), comparability ( $0-2$ points), and study outcomes ( $0-3$ points). Scores of 6 points or more were deemed to be of relatively high quality. 


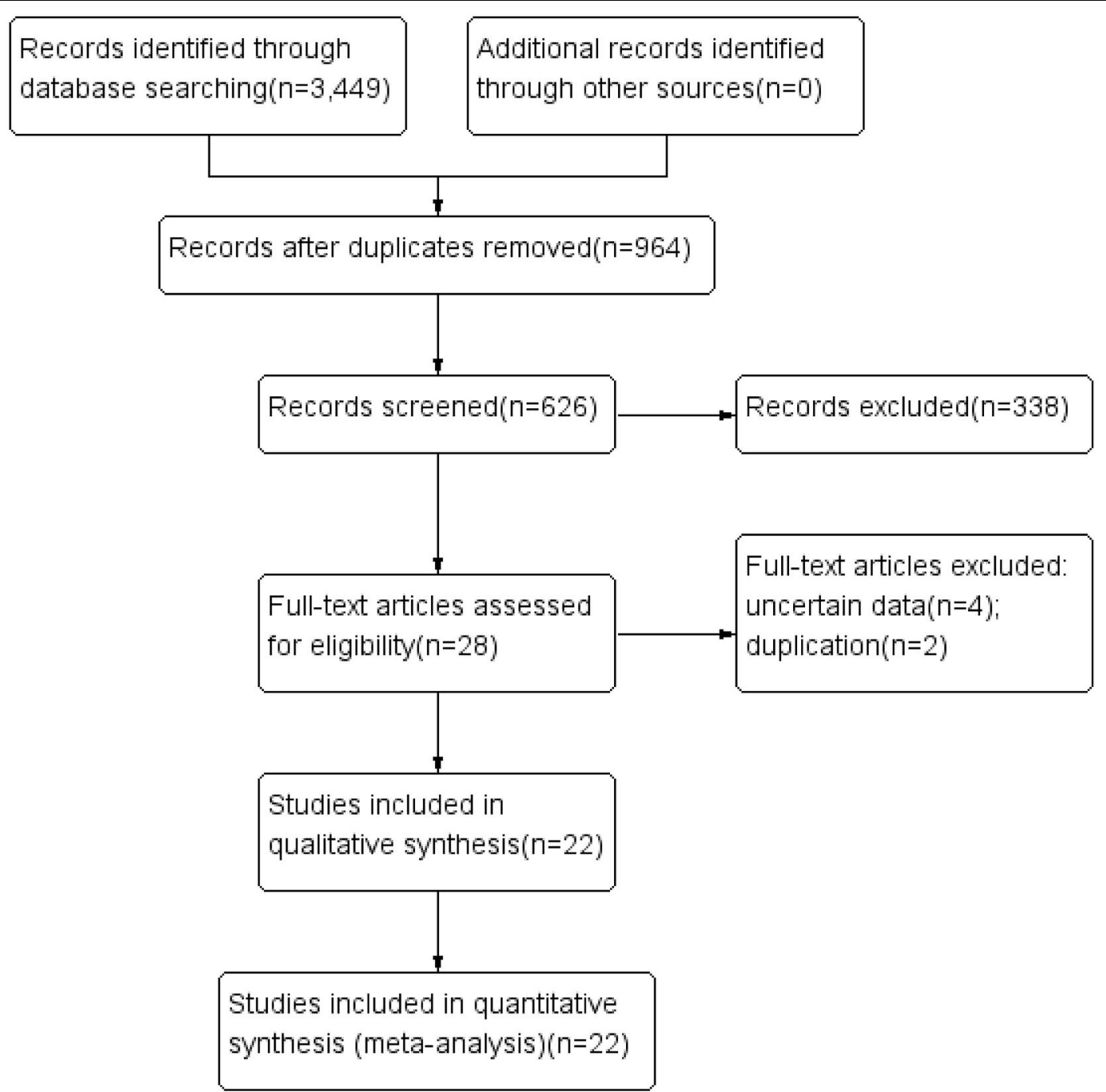

FIGURE 1 | Flow chart of the literature selection.

\section{Statistical Analysis}

All calculations and graphs were made using the Revman 5.3 software (The Nordic Cochrane Center, The Cochrane Collaboration, 2014). Time-to-event data (e.g., OS) were analyzed using the HRs. Chi-squared test was used to evaluate heterogeneity among studies, and $I^{2}$ was used to measure the magnitude of heterogeneity (9). Results with $P \geq 1$ and $I^{2} \leq 50 \%$ indicated a lack of significant heterogeneity; in such cases, the Mantel-Haenszel fixed-effects model was used for meta-analysis. Subgroup analysis was performed to evaluate the possible source of heterogeneity and to further study the preliminary results. If there was no obvious heterogeneity, the fixed model was used to estimate the pooled HR (10). Otherwise, the randomeffects model was used (11). Sensitivity analysis was performed to evaluate the risk of bias of studies. Publication bias was assessed by visually examining the funnel plots (12). A trimand-fill method was applied to estimate asymmetry in the funnel plot (13).

\section{RESULTS}

\section{Characteristics of the Studies}

A flow chart of literature selection is shown in Figure 1. A total of 3,449 articles were screened via a primary search of the literature database. After reading the titles and abstracts to remove irrelevant items, and reading the full texts to eliminate those that did not meet the inclusion criteria, we obtained 28 research reports that evaluated the OS of glioma patients treated with carmustine. Finally, four uncertain and two duplicate studies were excluded, and a total of 22 studies (9 RCTs and 13 cohort studies) involving 5,821 glioma patients were included in this meta-analysis $[6,14-34]$. The characteristics of the included studies are shown in Table 1. In the included literature, 19 studies were carried out in GBM patients, and two studies were in anaplastic glioma patients, and one study was in patients with gliomatosis. Six RCTs used a placebo-controlled, doubleblind, randomized method (14-19). In the other three RCTs, 
implantation of carmustine was not randomized and was decided according to clinical conditions $(6,20,21)$. The comparability of one cohort study is obscure (22), while that of others is relatively distinct (23-34). The results of the methodological quality assessment using NOS and Jadad scale are also shown in Table 1.

\section{Association Between Carmustine Treatment and Survival of Glioma Patients}

Meta-analysis of the included studies showed that glioma patients treated with carmustine had a better OS (HR $=0.85,95 \%$ $\mathrm{CI}=0.79-0.92, P<0.0001, I^{2}=45 \%$ ) (Figure 2A) and PFS $(\mathrm{HR}=0.85,95 \% \mathrm{CI}=0.77-0.94, P=0.002, I=67 \%)$ (Figure 2B) than non-carmustine-treated patients. Since the heterogeneity of survival analysis was high (OS 45\% and PFS 67\%), the correlation between carmustine administration and survival of glioma patients was further investigated in different subgroups in order to understand the possible source of heterogeneity and to decrease its interference.

It is well-known that the degree of malignancy is the most important factor for the prognosis of glioma patients (35). Therefore, the effect of carmustine on the OS of glioma patients with different pathological grades was first analyzed. Among the included studies, 15 evaluated the OS of GBM patients $(6,14,17-$ $23,26,27,29,33,34)$, and 2 evaluated the OS of anaplastic glioma patients $(16,30)$. Carmustine showed a positive benefit on the OS of $\mathrm{GBM}$ patients $(\mathrm{HR}=0.84,95 \% \mathrm{CI}=0.78-0.91, P<0.00001$, $I^{2}=27 \%$ ) (Figure 3A). However, there was no OS benefit from carmustine treatment in anaplastic glioma patients $(\mathrm{HR}=1.20$, 95\% CI $=0.70-2.07, P=0.50$ ) (Figure 3A).

The response to therapy is always different between newly diagnosed and recurrent GBM patients (23). Thus, we further evaluated the impact of carmustine on the OS of newly diagnosed and recurrent GBM patients in eight $(6,19,21-23,25,26,29)$, and four $(14,20,27,29)$ studies, respectively. This subgroup analysis showed that carmustine treatment contributed favorably to the OS of both newly diagnosed and recurrent GBM patients (newly diagnosed: $\mathrm{HR}=0.86,95 \% \mathrm{CI}=0.79-0.95, P=0.002, I^{2}=3 \%$; recurrent: $\left.\mathrm{HR}=0.77,95 \% \mathrm{CI}=0.67-0.89, P=0.0002, I^{2}=0 \%\right)$ (Figure 3B).

Next, we investigated whether the addition of carmustine therapy as a supplement to STUPP protocol could confer more significant survival benefits for GBM patients. There were only two studies that reported the comparison of survival benefits between patients with TMZ chemotherapy and TMZ plus carmustine chemotherapy $(25,33)$. The analysis of the two studies showed that GBM patients receiving combined chemotherapy (carmustine plus TMZ) had longer OS than those receiving $\mathrm{TMZ}$ alone $(\mathrm{HR}=0.78,95 \% \mathrm{CI}=0.63-0.97, P=0.02$, $\left.I^{2}=0 \%\right)$ (Figure 3C).

\section{Association Between the Administration Route of Carmustine and the Survival of GBM Patients}

Prior to the invention of Gliadel ${ }^{\circledR}$ wafers, carmustine was administered only by intravenous injection. We next examined whether the administration routes had an impact on the survival benefit of adjuvant carmustine chemotherapy in GBM patients. Carmustine was administered by intravenous injection in 6 studies $(16,17,22,24,25,29)$ and was implanted into the resection cavity in 12 studies $(6,14,17-21,23,26,33,34)$. Carmustine administrated by both routes prolonged the OS in GBM patients (resection cavity: $\mathrm{HR}=0.84,95 \% \mathrm{CI}=0.78-0.92$, $P<0.0001, I^{2}=39 \%$; intravenous: $\mathrm{HR}=0.86,95 \% \mathrm{CI}=0.75-$ $-0.99, P=0.04, I^{2}=69 \%$ ) (Figure 4A).

There is no recommended standard dose of carmustine wafer since the size of the tumor resection cavity varies among patients. Then, we further examined whether the survival benefit of carmustine was associated with the doses used in the studies. The cutoff for the higher dose of carmustine wafer was $\geq 8$ pieces and that for the lower dose was $\leq 7$ pieces. No statistical difference was observed between the two doses ( $\mathrm{HR}=0.65,95 \% \mathrm{CI}=0.38-1.10$, $P=0.11, I^{2}=0 \%$ ) (Figure 4B).

\section{Adverse Events}

Although there was no severe lethal toxicity, a clinically apparent pulmonary fibrosis caused by intravenous administration of carmustine, with a reported incidence of $0.6-5 \%$, should be considered. As a controversial therapeutic option, carmustine wafer implantation was also reported to result in several side effects. Patients receiving carmustine wafer implantation had higher rates of complications $(\mathrm{OR}=1.70$, $\left.95 \% \mathrm{CI}=1.08-2.67, P=0.02, I^{2}=15 \%\right)$ than the patients in the non-carmustine group (Supplementary Figure 1). The most common complication with carmustine wafer implantation was post-operative infection. Other adverse events included edema-related intracranial pressure change, healing defect, epileptic seizure, and neurological worsening.

\section{Publication Bias}

A funnel plot was used to measure the publication bias. The funnel plot of the improvement in performance status showed low potential publication bias in the included studies (Supplementary Figure 2).

\section{DISCUSSION}

Radiotherapy combined with concomitant and adjuvant TMZ after maximal safe resection, namely, STUPP protocol, has been widely adopted since 2005 for newly diagnosed GBM (36). Although systemic chemotherapy with TMZ is the most widely used regimen for glioma after tumor resection due to its well-tolerated and favorable clinical outcome, several review researches have reported that carmustine is also beneficial for glioma $(37,38)$. Previous reviews mainly discussed the survival benefit of carmustine in newly diagnosed GBM. In this study, more subgroup analyses, including glioma grade, newly diagnosed and recurrent GBM, administration routes, and doses, were also performed to comprehensively evaluate the benefits of carmustine in different subpopulation.

Significant OS and PFS benefits of carmustine treatment in glioma patients were observed in this study. The subgroup analysis showed that carmustine chemotherapy was associated 
TABLE 1 | Characteristics of included studies.

\begin{tabular}{|c|c|c|c|c|c|c|c|c|}
\hline Study & Year & District & $\begin{array}{l}\text { Patients } \\
\text { with/without } \\
\text { carmustine }\end{array}$ & $\begin{array}{l}\text { Adjuvant therapy (with/without } \\
\text { carmustine) }\end{array}$ & Study design & $\begin{array}{l}\text { Medication } \\
\text { method }\end{array}$ & Grade of glioma & $\begin{array}{l}\text { Quality of } \\
\text { studies(score) }\end{array}$ \\
\hline Affronti et al. (23) & 2009 & Occident & $36 / 49$ & $\begin{array}{l}\mathrm{RT} \text { and concurrent TMZ plus } \\
\text { rotational chemotherapy. }\end{array}$ & Cohort & Resection cavity & GBM & NOS (9) \\
\hline Autran et al. (24) & 2019 & France & $15 / 7$ & TMZ/Other treatment & Cohort & Intravenous & Gliomatosis & NOS (8) \\
\hline Brem et al. (14) & 1995 & USA & $72 / 73$ & RT and systemic chemotherapy & $\mathrm{RCT}$ & Resection cavity & GBM & Jadad (5) \\
\hline $\begin{array}{l}\text { Chaichana et al. } \\
\text { (25) }\end{array}$ & 2010 & USA & 148/192 & Adjuvant radiation and chemotherapy & Cohort & Intravenous & GBM & NOS (7) \\
\hline $\begin{array}{l}\text { Chaichana et al. } \\
\text { (26) }\end{array}$ & 2014 & USA & $64 / 195$ & $\mathrm{RT}$ and TMZ & Cohort & Resection cavity & GBM & NOS (8) \\
\hline \multirow[t]{2}{*}{ De Bonis et al. (15) } & 2012 & Italy & $10 / 67$ & Adjuvant therapy with TMZ & $\mathrm{RCT}$ & Resection cavity & GBM & Jadad (4) \\
\hline & 2012 & Italy & $17 / 71$ & Adjuvant therapy with TMZ & $\mathrm{RCT}$ & Resection cavity & GBM & Jadad (4) \\
\hline \multirow[t]{2}{*}{$\begin{array}{l}\text { Esquenazi et al. } \\
\text { (21) }\end{array}$} & 2017 & USA & $42 / 44$ & $\begin{array}{l}\text { Adjuvant radiotherapy and } \\
\text { concomitant TMZ therapy }\end{array}$ & Cohort & Resection cavity & GBM & $\operatorname{NOS}(9)$ \\
\hline & 2017 & USA & $42 / 44$ & $\begin{array}{l}\text { Adjuvant radiotherapy and } \\
\text { concomitant TMZ therapy }\end{array}$ & Cohort & Resection cavity & GBM & NOS (9) \\
\hline Jungk et al. (27) & 2016 & Germany & $34 / 29$ & RT and TMZ & Cohort & Intravenous & GBM & NOS (8) \\
\hline Kunwar et al. (28) & 2010 & Europe & $93 / 183$ & Adjuvant therapy & $\mathrm{RCT}$ & Resection cavity & GBM & Jadad (4) \\
\hline Loureiro et al. (20) & 2015 & Israel & $33 / 82$ & $\begin{array}{l}\text { RT and sequencing chemotherapy/ } \\
\text { RT concurrent with chemotherapy }\end{array}$ & Cohort & Intravenous & GBM & NOS (8) \\
\hline Louvel et al. (29) & 2016 & France & $254 / 438$ & $\begin{array}{l}\text { Standard combined } \\
\text { chemoradiotherapy }\end{array}$ & Cohort & Intravenous & GBM & NOS (8) \\
\hline McGirt et al. (30) & 2009 & USA & - & TMZ administered with radiotherapy & Cohort & Resection cavity & Anaplastic glioma & NOS (7) \\
\hline Pallud et al. (22) & 2015 & France & $354 / 433$ & Chemoradiation standard protocol & $\mathrm{RCT}$ & Resection cavity & GBM & Jadad (5) \\
\hline Pavlov et al. (31) & 2015 & France & $50 / 33$ & Stupp regimen & Cohort & Resection cavity & GBM & NOS (7) \\
\hline Price et al. (32) & 2012 & UK & 94/202 & Radiotherapy and Stupp protocol & Cohort & Resection cavity & GBM & NOS (6) \\
\hline Roux et al. (6) & 2017 & France & $123 / 217$ & $\begin{array}{l}\text { Standard combined } \\
\text { chemoradiotherapy }\end{array}$ & $\mathrm{RCT}$ & Resection cavity & GBM & Jadad (5) \\
\hline \multirow[t]{2}{*}{ Sage et al. (33) } & 2018 & UK & 78/182 & $\begin{array}{l}\text { Temozolomide-based } \\
\text { chemoradiotherapy protocol }\end{array}$ & Cohort & Resection cavity & GBM & NOS (8) \\
\hline & 2018 & UK & $76 / 76$ & $\begin{array}{l}\text { Temozolomide-based } \\
\text { chemoradiotherapy protocol }\end{array}$ & Cohort & Resection cavity & GBM & NOS (8) \\
\hline Schold et al. (16) & 1993 & Occident & $128 / 121$ & Radiotherapy & $\mathrm{RCT}$ & Intravenous & Anaplastic glioma & Jadad (3) \\
\hline \multirow[t]{2}{*}{ Sun et al. (17) } & 2015 & USA & $4 / 204$ & $\begin{array}{l}\text { Radiotherapy and concurrent } \\
\text { temozolomide chemotherapy }\end{array}$ & $\mathrm{RCT}$ & Intravenous & GBM & Jadad (4) \\
\hline & 2015 & USA & 9/199 & $\begin{array}{l}\text { Radiotherapy and concurrent } \\
\text { temozolomide chemotherapy }\end{array}$ & $\mathrm{RCT}$ & Resection cavity & GBM & Jadad (4) \\
\hline Valtonen et al. (18) & 1997 & Northern Europe & $5 / 1$ & Standard radiotherapy & RCT & Resection cavity & GBM & Jadad (4) \\
\hline Westphal et al. (19) & 2003 & Europe & $101 / 106$ & Radiotherapy & $\mathrm{RCT}$ & Resection cavity & GBM & Jadad (5) \\
\hline Zanello et al. (34) & 2017 & France & $194 / 583$ & $\begin{array}{l}\text { Standard combined } \\
\text { radiochemotherapy }\end{array}$ & Cohort & Resection cavity & GBM & NOS (8) \\
\hline
\end{tabular}


A

\begin{tabular}{l} 
Study or Subgroup \\
\hline Affronti, M. L 2009 \\
Autran, D 2019 \\
Brem, H 1995 \\
Chaichana, K 2010 \\
Chaichana, K. L 2014 \\
Esquenazi, Y(A) 2017 \\
Esquenazi, Y(B) 2017 \\
Kunwar, S 2010 \\
Loureiro, L V 2015 \\
Louvel, G 2016 \\
McGirt, M 2009 \\
Pallud, J 2015 \\
Roux, A 2017 \\
Sage, W(A) 2018 \\
Sage, W(B) 2018 \\
Schold, S. C., Jr 1993 \\
Sun, M. Z(A) 2015 \\
Sun, M. Z(B) 2015 \\
Valtonen, S 1997 \\
Westphal, M 2003 \\
Zanello, M 2017
\end{tabular}

Total $(95 \% \mathrm{Cl})$

Heterogeneity: $\mathrm{Chi}^{2}=36.08, \mathrm{df}=20(\mathrm{P}=0.02) ; \mathrm{I}^{2}=45 \%$

Test for overall effect: $Z=4.32(P<0.0001)$
Hazard Ratio Hazard Ratio

log[Hazard Ratio] SE Weight IV, Fixed, 95\% Cl

$\begin{array}{llll}-0.2877 & 0.2494 & 2.2 \% & 0.75\end{array}[0.46,1.22]$

$\begin{array}{llll}1.8924 & 0.6149 & 0.4 \% & 6.64[1.99,22.14]\end{array}$

$\begin{array}{llll}-0.4005 & 0.1702 & 4.7 \% & 0.67[0.48,0.94]\end{array}$

$\begin{array}{llll}-0.2627 & 0.1232 & 8.9 \% & 0.77[0.60,0.98]\end{array}$

$\begin{array}{llll}0.044 & 0.1578 & 5.4 \% & 1.04[0.77,1.42]\end{array}$

$\begin{array}{llll}0.0583 & 0.2574 & 2.0 \% & 1.06[0.64,1.76]\end{array}$

$\begin{array}{llll}-0.0408 & 0.2571 & 2.0 \% & 0.96[0.58,1.59]\end{array}$

$\begin{array}{llll}-0.1165 & 0.1449 & 6.4 \% & 0.89\end{array}[0.67,1.18]$

$\begin{array}{llll}0.1689 & 0.3052 & 1.5 \% & 1.18[0.65,2.15]\end{array}$

$\begin{array}{llll}-0.1625 & 0.0918 & 16.0 \% & 0.85[0.71,1.02]\end{array}$

$\begin{array}{llll}0.1931 & 0.2903 & 1.6 \% & 1.21[0.69,2.14]\end{array}$

$\begin{array}{llll}-0.0513 & 0.0877 & 17.6 \% & 0.95[0.80,1.13]\end{array}$

$\begin{array}{llll}-0.3711 & 0.1746 & 4.4 \% & 0.69[0.49,0.97]\end{array}$

$\begin{array}{llll}-0.2107 & 0.2069 & 3.2 \% & 0.81\end{array}[0.54,1.22]$

$\begin{array}{llll}0.0953 & 0.1689 & 4.7 \% & 1.10[0.79,1.53]\end{array}$

$\begin{array}{llll}0.1026 & 0.9387 & 0.2 \% & 1.11[0.18,6.98]\end{array}$

$\begin{array}{llll}-0.3299 & 0.7829 & 0.2 \% & 0.72[0.15,3.34]\end{array}$

$\begin{array}{llll}0.6109 & 0.6819 & 0.3 \% & 1.84[0.48,7.01]\end{array}$

$\begin{array}{llll}-1.3093 & 0.4581 & 0.6 \% & 0.27\end{array}[0.11,0.66]$

$\begin{array}{llll}-0.2744 & 0.165 & 5.0 \% & 0.76[0.55,1.05]\end{array}$

$\begin{array}{llll}-0.3425 & 0.1032 & 12.7 \% & 0.71\end{array}[0.58,0.87]$

$100.0 \% \quad 0.85[0.79,0.92]$

IV. Fixed, 95\% Cl

B

\begin{tabular}{l} 
Study or Subgroup \\
\hline Autran, D 2019 \\
Jungk, C 2016 \\
Louvel, G 2016 \\
Pallud, J 2015 \\
Roux, A 2017 \\
Schold, S. C., Jr 1993 \\
Sun, M. Z(A) 2015 \\
Sun, M. Z(B) 2015
\end{tabular}

Total $(95 \% \mathrm{Cl})$

Heterogeneity: $\mathrm{Chi}^{2}=21.17, \mathrm{df}=7(\mathrm{P}=0.004) ; \mathrm{I}^{2}=67 \%$

Test for overall effect: $Z=3.09(P=0.002)$

log[Hazard Ratio] SE Weight IV. Fixed, 95\% C

$\begin{array}{llll}1.3517 & 0.5611 & 0.8 \% & 3.86\end{array}[1.29,11.60]$

$\begin{array}{llll}-0.734 & 0.3128 & 2.7 \% & 0.48[0.26,0.89]\end{array}$

$\begin{array}{llll}-0.2107 & 0.0893 & 32.7 \% & 0.81[0.68,0.96]\end{array}$

$\begin{array}{llll}-0.1985 & 0.0807 & 40.0 \% & 0.82[0.70,0.96]\end{array}$

$-0.3011 \quad 0.1514 \quad 11.4 \% \quad 0.74[0.55,1.00]$

$\begin{array}{llll}0.2469 & 0.1501 & 11.6 \% & 1.28\end{array}[0.95,1.72]$

$\begin{array}{llll}0.3192 & 0.7239 & 0.5 \% & 1.38[0.33,5.69]\end{array}$

$\begin{array}{llll}0.822 & 0.8476 & 0.4 \% & 2.28\end{array}[0.43,11.98]$

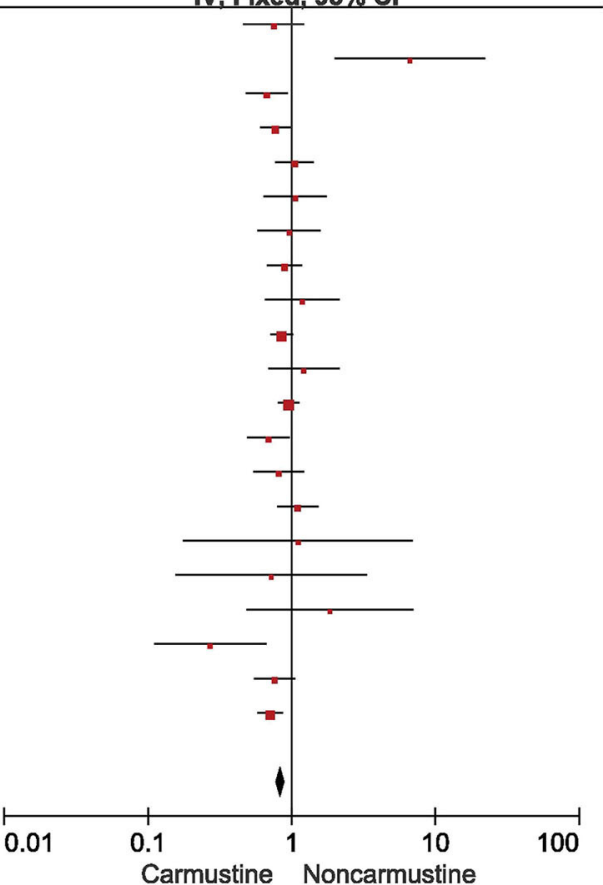

$100.0 \% \quad 0.85[0.77,0.94]$

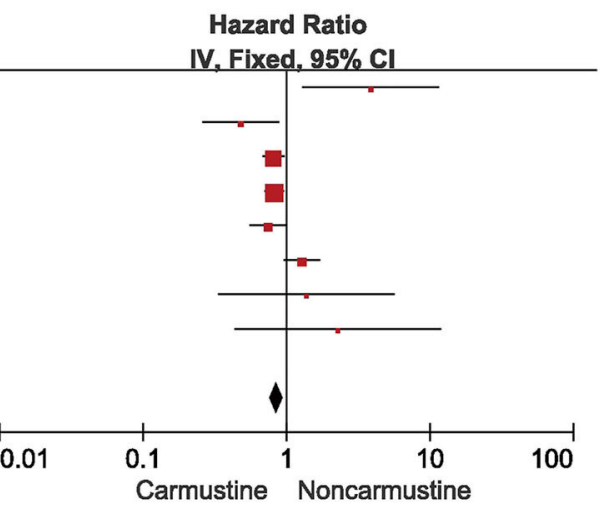

FIGURE 2 | Hazard ratios and 95\% confidence intervals of the association between carmustine implantation and overall survival (A) and progression-free survival (B) of glioma patients. $\mathrm{Cl}$, confidence interval; df, degrees of freedom; SE, standard error.

with better OS in GBM patients but not in anaplastic glioma patients. More importantly, both newly diagnosed and recurrent GBM patients may benefit from carmustine treatment. It is noted that only two included studies reported the survival outcome in anaplastic glioma patients with or without carmustine chemotherapy. It is also reported that implantation of carmustine resulted in worse OS of patients with leptomeningeal gliomatosis (24). Therefore, it is likely that carmustine may be a reasonable option for patients with GBM but not for those with anaplastic glioma and low-grade glioma. Similar to carmustine, lomustine is always used as a combining chemotherapy agent with procarbazine and vincristine, which is commonly named PCV protocol. PCV regimen was reported to have a similar benefit on OS in patients with anaplastic astrocytoma, and GBM was similar to carmustine $(39,40)$, while another study showed that PCV was more effective than carmustine in anaplastic astrocytoma patients (41).

Historically, the nitrosourea derivate, carmustine, has been administered intravenously for glioma patients and, lately, has experienced a renaissance in Europe since the invention of biodegradable carmustine wafer. Carmustine administration, along with other adjuvant treatments, is being more frequently utilized because it not only improves OS and PFS but also has been well-tolerated for several years $(18,19)$. The effectiveness of carmustine wafer was evaluated by RCTs, and the result showed that it could significantly increase OS by 2-4 months in newly diagnosed GBM patients $(18,19)$. The prognostic advantage of carmustine wafer implantation on OS was also confirmed 


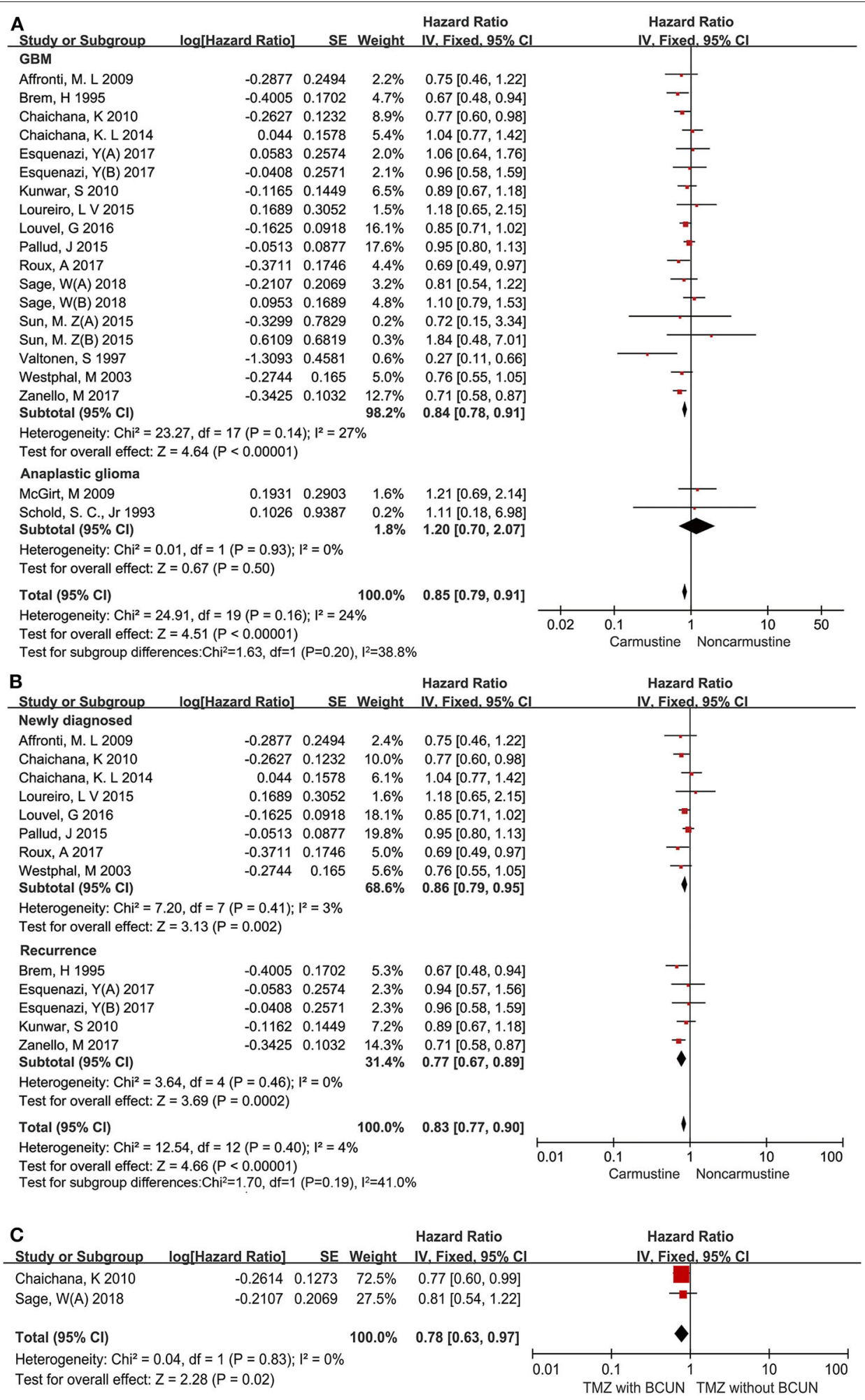

FIGURE 3 | Hazard ratios and 95\% confidence intervals of the association between carmustine implantation and overall survival in patients with different pathological grades of glioma (A), in newly diagnosed and recurrent GBM (B), and in patients with TMZ chemotherapy and TMZ plus carmustine chemotherapy (C). Cl, confidence interval; df, degrees of freedom; SE, standard error. 


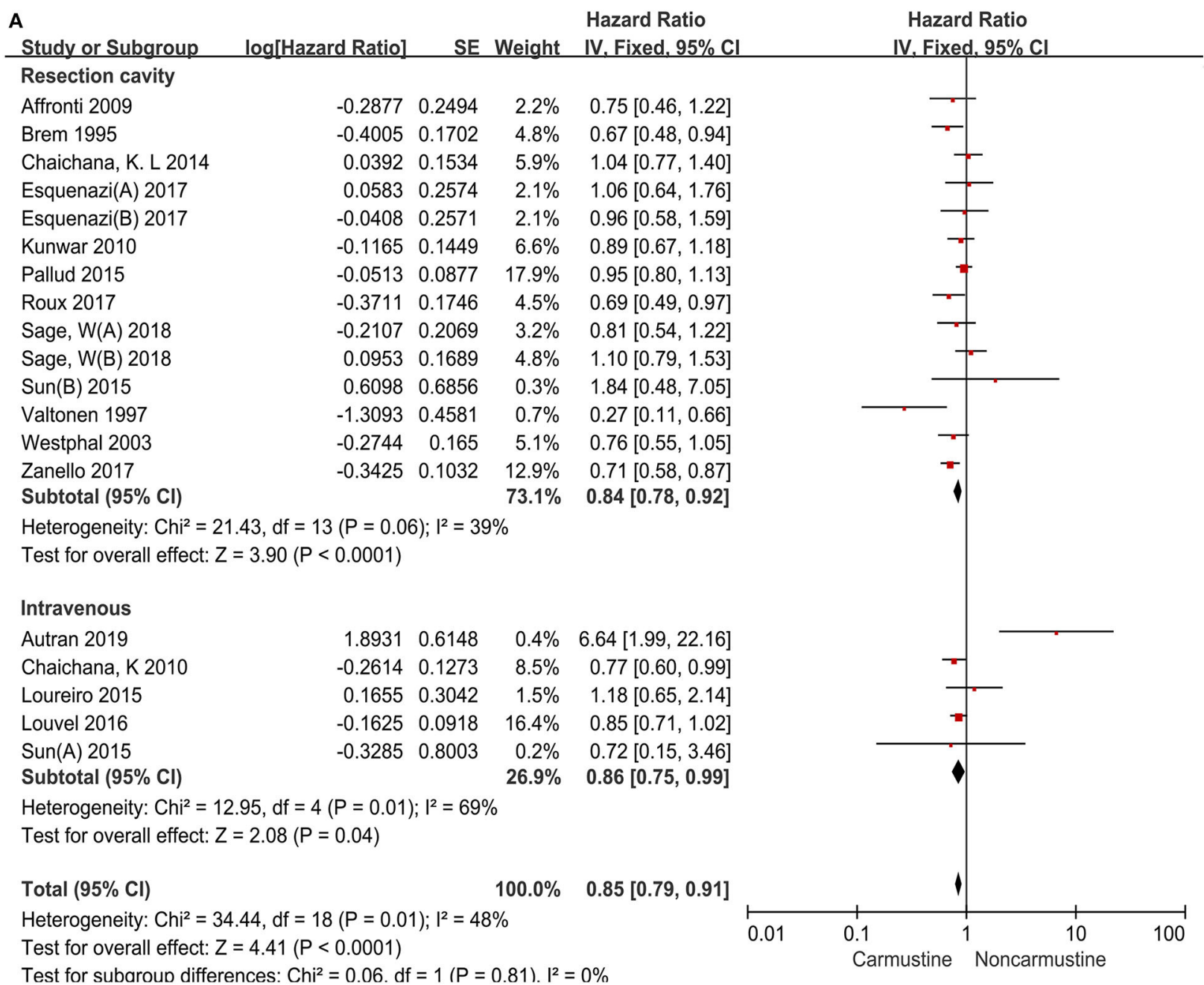

Test for subaroun differences: $\mathrm{Chi}^{2}=0.06 . \mathrm{df}=1(\mathrm{P}=0.81) . \mathrm{I}^{2}=0 \%$

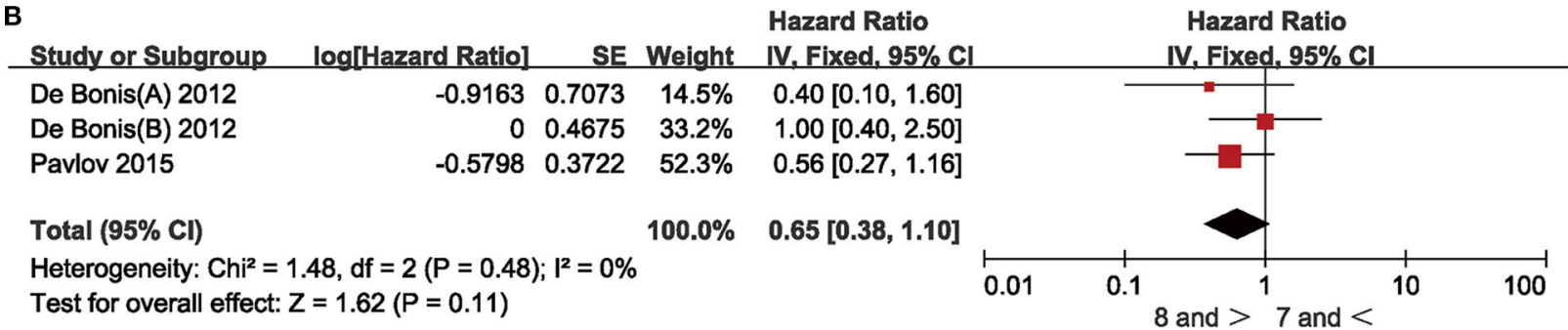

FIGURE 4 | Hazard ratios and 95\% confidence intervals of the association between overall survival and administration routes of carmustine (A), and different doses of carmustine wafer (B) in GBM patients. Cl, confidence interval; df, degrees of freedom; SE, standard error.

independent of the extent of resection and the Karnofsky performance score (KPS) (6). This suggested an additional survival benefit of carmustine wafer implantation together with subtotal or total surgical resection at the first surgery of newly diagnosed GBM patients (6). Our study showed that both intravenous administration and carmustine wafer implantation into the resection cavity were associated with better OS in GBM patients. Another published meta-analysis also demonstrated that the median survival time of newly diagnosed high-grade glioma patients who received carmustine wafer treatment was 16 months, and the 1- and 2-year overall survival rates were 67 and $26 \%$, respectively (42). The dose of carmustine wafer is always dependent on the size of the tumor resection cavity, which varies among patients. Therefore, the effect of different doses of carmustine wafer on OS was evaluated, and no statistical difference was observed in our study. In the seven included 
studies, intravenous carmustine was given $100 \mathrm{mg} / \mathrm{m}^{2}$ on day 1 and day 2 or $200 \mathrm{mg} / \mathrm{m}^{2}$ on day 1 and repeated every 6-8 weeks. Due to the insufficiency of survival data in the included studies, the correlation between the intravenous dose and the survival outcome was not further examined. In fact, previous studies have demonstrated that longer carmustine exposure duration and lower toxicity could be provided by polymer-based wafer delivery rather than intravenous injection (43).

Although the efficacy of carmustine for GBM is established from our meta-analysis and other seminal clinical trial results, its safety remains a matter of debate. Jungk et al. performed a retrospective analysis on the side effects of carmustine-based chemotherapy in 163 recurrent glioma patients who accepted an intravenous administration of freshly prepared carmustine (28). The results showed that carmustine was well-tolerated with predominantly mild side effects, although $54 \%$ of the patients experienced carmustine-related side effects. Interestingly, side effects were not observed equally among tumor grades, with WHO grade IV patients experiencing them least frequently, and WHO grade II patients experiencing them most frequently. The most common adverse event was post-operative infections with carmustine wafer implantation.

As no RCTS or cohort studies comparing the benefits of carmustine alone with TMZ alone were enrolled in our meta-analysis according to the included criteria, we performed the analysis with two published retrospective studies (44, 45) to evaluate the efficacy of carmustine and TMZ. The better efficacy of TMZ alone than carmustine alone was observed in newly diagnosed GBM patients ( $\mathrm{HR}=0.65,95 \%$ $\mathrm{CI}=0.44-0.95, P=0.03, I^{2}=0 \%$ ) (Supplementary Figure 3). However, carmustine wafer implantation may offer a bridge of "non-therapeutic" period between surgical resection and onset of radiochemotherapy. Our analysis showed that combining carmustine plus TMZ conferred a better OS than TMZ alone, indicating that carmustine wafer may be a supplement to standard STUPP protocol for GBM by offering a bridge of "nontherapeutic" period, allowing continuous chemotherapy. The effectiveness and safety of carmustine as a supplement to STUPP protocol need to be further investigated.

Although TMZ treatment is widely recommended due to its high efficacy, some GBM patients are not sensitive to TMZ therapy, especially those with non-methylated O-6-methylguanine-DNA methyltransferase (MGMT) (46). The status of MGMT promoter methylation is associated with tumor response to TMZ therapy (46). Our previous study demonstrated the universal predictive value of $M G M T$ methylation in newly diagnosed, elderly, and recurrent GBM patients (46). Although the efficacy of carmustine is less than that of TMZ, carmustine may be a reasonable option for GBM patients with non-methylated MGMT promoter. Cardona et al. reported that following carmustine/bevacizumab treatment,

\section{REFERENCES}

1. Omuro A, DeAngelis LM. Glioblastoma and other malignant gliomas: a clinical review. JAMA. (2013) 310:1842-50. doi: 10.1001/jama.2013.280319 patients with a combination of an IDH mutation plus MGMT methylation had better OS and PFS than those with only one of these characteristics or those with none (47). Predictive molecular biomarkers for carmustine efficacy need to be further investigated.

\section{Limitations}

The interpretation of the present results should be considered under some limitations. First, the Karnofsky performance score is a crucial factor for deciding glioma treatment choices; however, there are few studies that have calculated HR for KPS. Therefore, we were unable to evaluate the association between KPS and carmustine administration. Second, the relationship between carmustine administration and important prognostic factors, including the extent of tumor resection and tumor molecular features, was not analyzed. We also did not assess the differences in response to BCNU therapy based on sex and ethnicity. Third, we focused on the effectiveness of carmustine alone, while there may be other adjuvant therapies influencing the results as well.

\section{CONCLUSION}

Carmustine implantation in resection cavity provides survival benefit for GBM patients, and it may be a promising supplement to standard therapeutic protocol by offering a bridge between surgical resection and onset of TMZ therapy.

\section{AUTHOR CONTRIBUTIONS}

Z-ZX and Z-FW collected the data, performed the statistical analysis, and drafted the report. Y-HZ participated in data collection analysis. TL, W-HH, and CM participated in the interpretation of the data and critically reviewed the manuscript. Z-QL designed the study and contributed to the interpretation and discussion of the results. All authors read and approved the final manuscript. All authors contributed to the article and approved the submitted version.

\section{FUNDING}

This research was supported by grants from the National Natural Science Foundation of China (no. 81573459), National Health Commission of China (2018ZX-07S-011), and Medical Science Advancement Program of Wuhan University (no. TFJC2018003).

\section{SUPPLEMENTARY MATERIAL}

The Supplementary Material for this article can be found online at: https://www.frontiersin.org/articles/10.3389/fneur. 2020.01036/full\#supplementary-material

2. Thakkar JP, Dolecek TA, Horbinski C, Ostrom QT, Lightner DD, Barnholtz-Sloan JS, et al. Epidemiologic and molecular prognostic review of glioblastoma. Cancer Epidemiol Biomarkers Prev. (2014) 23:198596. doi: 10.1158/1055-9965.EPI-14-0275 
3. Zhao Y-H, Wang Z-F, Pan Z-Y, Péus D, Delgado-Fernandez J, Pallud J, et al. A meta-analysis of survival outcomes following reoperation in recurrent glioblastoma: time to consider the timing of reoperation. Front Neurol. (2019) 10:286. doi: 10.3389/fneur.2019.00286

4. Lillehei KO, Kalkanis SN, Liau LM, Mydland DE, Olson J, Paleologos $\mathrm{NA}$, et al. Rationale and design of the 500-patient, 3-year, and prospective Vigilant ObservatIon of GlIadeL WAfer ImplaNT registry. CNS Oncol. (2018) 7:CNS08. doi: 10.2217/cns-2017-0036

5. Brem H, Mahaley MS, Vick NA, Black KL, Schold SC, Burger $\mathrm{PC}$, et al. Interstitial chemotherapy with drug polymer implants for the treatment of recurrent gliomas. J Neurosurg. (1991) 74:441-6. doi: 10.3171/jns.1991.74.3.0441

6. Roux A, Peeters S, Zanello M, Bou Nassif R, Abi Lahoud G, Dezamis E, et al. Extent of resection and Carmustine wafer implantation safely improve survival in patients with a newly diagnosed glioblastoma: a single center experience of the current practice. J Neurooncol. (2017) 135:8392. doi: 10.1007/s11060-017-2551-4

7. Jadad AR, Moore RA, Carroll D, Jenkinson C, Reynolds DJ, Gavaghan DJ, et al. Assessing the quality of reports of randomized clinical trials: is blinding necessary? Control Clin Trials. (1996) 17:1-12. doi: 10.1016/0197-2456(95)00134-4

8. Wells G, Shea B, O'Connell D, Peterson J, Welch V, Losos M, et al. The Newcastle-Ottawa Scale (NOS) for assessing the quality of non-randomized studies in meta-analysis. (2000).

9. Higgins JPT, Thompson SG. Quantifying heterogeneity in a meta-analysis. Stat Med. (2002) 21:1539-58. doi: 10.1002/sim.1186

10. Adejumo AO, Adetunji, AA. Cochran-Mantel-Haenszel test for repeated tests of independence: an application in examining students' performance. J Educ Pract. (2013) 4

11. DerSimonian R, Laird N. Meta-analysis in clinical trials. Control Clin Trials. (1986) 7:177-88. doi: 10.1016/0197-2456(86)90046-2

12. Begg CB, Mazumdar M. Operating characteristics of a rank correlation test for publication bias. Biometrics. (1994) 50:1088-101. doi: 10.2307/2533446

13. Duval S, Tweedie R. Trim and fill: a simple funnel-plot-based method of testing and adjusting for publication bias in meta-analysis. Biometrics. (2000) 56:455-63. doi: 10.1111/j.0006-341X.2000.00455.x

14. Brem H, Piantadosi S, Burger PC, Walker M, Selker R, Vick NA, et al. Placebo-controlled trial of safety and efficacy of intraoperative controlled delivery by biodegradable polymers of chemotherapy for recurrent gliomas. The Polymer-brain Tumor Treatment Group. Lancet. (1995) 345:100812. doi: 10.1016/S0140-6736(95)90755-6

15. De Bonis P, Anile C, Pompucci A, Fiorentino A, Balducci M, Chiesa S, et al. Safety and efficacy of Gliadel wafers for newly diagnosed and recurrent glioblastoma. Acta Neurochir. (2012) 154:1371-78. doi: 10.1007/s00701-012-1413-2

16. Schold SC, Herndon JE, Burger PC, Halperin EC, Vick NA, Cairncross $\mathrm{JG}$, et al. Randomized comparison of diaziquone and carmustine in the treatment of adults with anaplastic glioma. J Clin Oncol. (1993) 11:7783. doi: 10.1200/JCO.1993.11.1.77

17. Sun MZ, Oh T, Ivan ME, Clark AJ, Safaee M, Sayegh ET, et al. Survival impact of time to initiation of chemoradiotherapy after resection of newly diagnosed glioblastoma. J Neurosurg. (2015) 122:1144-50. doi: 10.3171/2014.9.JNS14193

18. Valtonen S, Timonen U, Toivanen P, Kalimo H, Kivipelto L, Heiskanen O, et al. Interstitial chemotherapy with carmustine-loaded polymers for highgrade gliomas: a randomized double-blind study. Neurosurgery. (1997) 41:449. doi: 10.1097/00006123-199707000-00011

19. Westphal M, Hilt DC, Bortey E, Delavault P, Olivares R, Warnke PC, et al. A phase 3 trial of local chemotherapy with biodegradable carmustine (BCNU) wafers (Gliadel wafers) in patients with primary malignant glioma. Neuro Oncol. (2003) 5:79-88. doi: 10.1093/neuonc/5.2.79

20. Loureiro LVM, Pontes LdeB, Callegaro-Filho D, Koch LdeO, Weltman E, Victor EdaS, et al. Waiting time to radiotherapy as a prognostic factor for glioblastoma patients in a scenario of medical disparities. Arq Neuropsiquiatr. (2015) 73:104-10. doi: 10.1590/0004-282X20140202

21. Esquenazi Y, Friedman E, Liu Z, Zhu J-J, Hsu S, Tandon N. The survival advantage of "supratotal" resection of glioblastoma using selective cortical mapping and the subpial technique. Neurosurgery. (2017) 81:404. doi: 10.1093/neuros/nyx359
22. Pallud J, Audureau E, Noel G, Corns R, Lechapt-Zalcman E, Duntze $\mathrm{J}$, et al. Long-term results of carmustine wafer implantation for newly diagnosed glioblastomas: a controlled propensity-matched analysis of a French multicenter cohort. Neuro Oncol. (2015) 17:1609-19. doi: 10.1093/neuonc/nov126

23. Affronti ML, Heery CR, Herndon JE, Rich JN, Reardon DA, Desjardins A, et al. Overall survival of newly diagnosed glioblastoma patients receiving carmustine wafers followed by radiation and concurrent temozolomide plus rotational multiagent chemotherapy. Cancer. (2009) 115:3501-11. doi: 10.1002/cncr.24398

24. Autran D, Barrie M, Matta M, Monserrat C, Campello C, Petrirena G, et al. Leptomeningeal gliomatosis: a single institution study of 31 patients. Anticancer Res. (2019) 39:1035-41. doi: 10.21873/anticanres.13210

25. Chaichana K, Parker S, Olivi A, Quiñones-Hinojosa A. A proposed classification system that projects outcomes based on preoperative variables for adult patients with glioblastoma multiforme. J Neurosurg. (2010) 112:9971004. doi: $10.3171 / 2009$.9.JNS09805

26. Chaichana KL, Jusue-Torres I, Navarro-Ramirez R, Raza SM, PascualGallego M, Ibrahim A, et al. Establishing percent resection and residual volume thresholds affecting survival and recurrence for patients with newly diagnosed intracranial glioblastoma. Neuro Oncol. (2014) 16:11322. doi: $10.1093 /$ neuonc/not137

27. Jungk C, Chatziaslanidou D, Ahmadi R, Capper D, Bermejo JL, Exner J, et al. Chemotherapy with BCNU in recurrent glioma: analysis of clinical outcome and side effects in chemotherapy-naïve patients. BMC Cancer. (2016) 16:81. doi: 10.1186/s12885-016-2131-6

28. Kunwar S, Chang S, Westphal M, Vogelbaum M, Sampson J, Barnett $\mathrm{G}$, et al. Phase III randomized trial of CED of IL13-PE38QQR vs Gliadel wafers for recurrent glioblastoma. Neuro Oncol. (2010) 12:87181. doi: 10.1093/neuonc/nop054

29. Louvel G, Metellus P, Noel G, Peeters S, Guyotat J, Duntze J, et al. Delaying standard combined chemoradiotherapy after surgical resection does not impact survival in newly diagnosed glioblastoma patients. Radiother Oncol. (2016) 118:9-15. doi: 10.1016/j.radonc.2016.01.001

30. McGirt MJ, Chaichana KL, Gathinji M, Attenello FJ, Than K, Olivi A, et al. Independent association of extent of resection with survival in patients with malignant brain astrocytoma. J Neurosurg. (2009) 110:15662. doi: $10.3171 / 2008.4 .17536$

31. Pavlov V, Page P, Abi-Lahoud G, Nataf F, Dezamis E, Robin A, et al. Combining intraoperative carmustine wafers and Stupp regimen in multimodal firstline treatment of primary glioblastomas. Br J Neurosurg. (2015) 29:52431. doi: 10.3109/02688697.2015.1012051

32. Price SJ, Whittle IR, Ashkan K, Grundy P, Cruickshank G. NICE guidance on the use of carmustine wafers in high grade gliomas: a national study on variation in practice. Br J Neurosurg. (2012) 26:3315. doi: 10.3109/02688697.2012.673651

33. Sage W, Guilfoyle M, Luney C, Young A, Sinha R, Sgubin D, et al. Local alkylating chemotherapy applied immediately after 5-ALA guided resection of glioblastoma does not provide additional benefit. J Neurooncol. (2018) 136:273-80. doi: 10.1007/s11060-017-2649-8

34. Zanello M, Roux A, Ursu R, Peeters S, Bauchet L, Noel G, et al. Recurrent glioblastomas in the elderly after maximal first-line treatment: does preserved overall condition warrant a maximal second-line treatment? J Neurooncol. (2017) 135:285-97. doi: 10.1007/s11060-017-2573-y

35. Gao F, Cui Y, Jiang H, Sui D, Wang Y, Jiang Z, et al. Circulating tumor cell is a common property of brain glioma and promotes the monitoring system. Oncotarget. (2016) 7:71330-40. doi: 10.18632/oncotarget.11114

36. Stupp R, Mason WP, van den Bent MJ, Weller M, Fisher B, Taphoorn $\mathrm{MJB}$, et al. Radiotherapy plus concomitant and adjuvant temozolomide for glioblastoma. N Engl J Med. (2005) 352:987-96. doi: 10.1056/NEJMoa043330

37. Garside R, Pitt M, Anderson R, Rogers G, Dyer M, Mealing S, et al. The effectiveness and cost-effectiveness of carmustine implants and temozolomide for the treatment of newly diagnosed high-grade glioma: a systematic review and economic evaluation. Health Technol Assess. (2007) 11:iii221. doi: 10.3310/hta11450

38. Xing W-k, Shao C, Qi Z-y, Yang C, Wang Z. The role of Gliadel wafers in the treatment of newly diagnosed GBM: a meta-analysis. Drug Des Devel Ther. (2015) 9:3341-8. doi: 10.2147/DDDT.S85943 
39. Prados MD, Scott C, Curran WJ, Nelson DF, Leibel S, Kramer S. Procarbazine, lomustine, and vincristine (PCV) chemotherapy for anaplastic astrocytoma: a retrospective review of radiation therapy oncology group protocols comparing survival with carmustine or PCV adjuvant chemotherapy. J Clin Oncol. (1999) 17:3389-95. doi: 10.1200/JCO.1999.17.11.3389

40. Cairncross JG, Macdonald DR. Successful chemotherapy for recurrent malignant oligodendroglioma. Ann Neurol. (1988) 23:360-4. doi: 10.1002/ana.410230408

41. Levin VA, Silver P, Hannigan J, Wara WM, Gutin PH, Davis RL, et al. Superiority of post-radiotherapy adjuvant chemotherapy with CCNU, procarbazine, and vincristine (PCV) over BCNU for anaplastic gliomas: NCOG 6G61 final report. Int J Radiat Oncol Biol Phys. (1990) 18:3214. doi: 10.1016/0360-3016(90)90096-3

42. Chowdhary SA, Ryken T, Newton HB. Survival outcomes and safety of carmustine wafers in the treatment of high-grade gliomas: a meta-analysis. J Neurooncol. (2015) 122:367-82. doi: 10.1007/s11060-015-1724-2

43. Wang CC, Li J, Teo CS, Lee T. The delivery of BCNU to brain tumors. J Control Release. (1999) 61:21-41. doi: 10.1016/S0168-3659(99) 00098-X

44. Illic R, Somma T, Savic D, Frio F, Milicevic M, Solari D, et al. A survival analysis with identification of prognostic factors in a series of 110 patients with newly diagnosed glioblastoma before and after introduction of the stupp regimen: a single-center observational study. World Neurosurg. (2017) 104:581-8. doi: 10.1016/j.wneu.2017.05.018
45. Vinjamuri M, Adumala RR, Altaha R, Hobbs GR, Crowell EB. Comparative analysis of temozolomide (TMZ) versus 1,3-bis (2-chloroethyl)-1 nitrosourea (BCNU) in newly diagnosed glioblastoma multiforme (GBM) patients. $J$ Neurooncol. (2009) 91:221-5. doi: 10.1007/s11060-008-9702-6

46. Zhao Y-H, Wang Z-F, Cao C-J, Weng H, Xu C-S, Li K, et al. The clinical significance of O-methylguanine-DNA methyltransferase promoter methylation status in adult patients with glioblastoma: a meta-analysis. Front Neurol. (2018) 9:127. doi: 10.3389/fneur.2018.00127

47. Cardona AF, Rojas L, Wills B, Ruiz-Patiño A, Abril L, Hakim F, et al. A comprehensive analysis of factors related to carmustine/bevacizumab response in recurrent glioblastoma. Clin Transl Oncol. (2019) 21:136473. doi: 10.1007/s12094-019-02066-2

Conflict of Interest: The authors declare that the research was conducted in the absence of any commercial or financial relationships that could be construed as a potential conflict of interest.

Copyright (C) 2020 Xiao, Wang, Lan, Huang, Zhao, Ma and Li. This is an open-access article distributed under the terms of the Creative Commons Attribution License (CC $B Y)$. The use, distribution or reproduction in other forums is permitted, provided the original author(s) and the copyright owner(s) are credited and that the original publication in this journal is cited, in accordance with accepted academic practice. No use, distribution or reproduction is permitted which does not comply with these terms. 